\title{
Dirac's hidden geometry
}

\author{
Paul Dirac insisted that his approach to quantum physics was geometric not algebraic. But where \\ is the evidence of this in his pioneering, algebra-rich papers?
}

\section{Graham Farmelo}

Of the few authentic visionaries modern science has known, Paul Dirac was the most inscrutable. He was a man of legendary quietness and privacy. His peers were bemused by his strikingly unusual way of looking at the world, while also amazed by the fecundity of his methods. Beginning in 1925, Dirac spent eight years developing quantum mechanics in a series of elegant papers that repeatedly took theoretical physicists by surprise. What, they wondered, lay behind Dirac's unique methods?

Butcolleagues seeking clues about the provenance of Dirac's ideas found none in his papers. He began each one with a deceptively straightforward, equationless introduction, before steam hammering his way through the mathematics, giving no quarter either to the faint hearted or to pedants.

Few of his papers contained a diagram and none offered any solace to readers trying to visualize what was represented by the blizzard of abstractions. Dirac would respond to requests to express his quantum reasoning in words or pictures by impassively shaking his head. So it is hardly surprising that Dirac's peers perceived him to be, above all, a brilliant algebraist extremely adept at manipulating abstract symbols, but uninterested in visualization.

Yet Dirac insisted that he was not primarily an algebraic theoretician. This insistence first emerged almost 40 years after his first quantum papers were published, during an interview with the historian of science Thomas Kuhn in the spring of 1963. Dirac declared to Kuhn that his approach was fundamentally geometrical and that he was "just no good" at doing "masses of algebraic calculations without picturing what the equation means".

Robert Oppenheimer, Dirac's close friend and admirer, was incredulous when he heard this. Oppenheimer had hardly ever seen Dirac draw a diagram but had always been awed by his algebraic skill. No, Oppenheimer assured Kuhn, Dirac was "principally algebraic".

Indeed, Oppenheimer went further and commented that Dirac's approach to physics was intuitive, like the mumbling savant Niels Bohr, although the two had completely different ways of communicating. "Bohr regarded mathematics as Dirac regards words, namely as a way to make himself intelligible to other people, which he hardly needs," he said.

In a scientific memoir nine years later, Dirac divided mathematicians cleanly into algebraists and geometers (the field of analysis apparently did not count). He had observed that mathematicians with a European training tended to be interested in geometry, following the school of the ancient Greeks, whereas those from an Asian background preferred algebra, which reference in Werner Heisenberg's pioneering paper to the existence of pairs of quantities with the strange property that $A \times B$ is not always equal to $B \times A$. Darrigol points out that this type of relationship occurs in the elementary theorems of basic projective geometry, which Dirac had certainly studied. So Dirac would have been ready to capitalize on the analogy and to develop a geometric picture of Heisenberg's theory. An intriguing explanation, but is it correct?

Dirac had a perfect opportunity to darify the point at a talk he gave in the autumn of 1972 at Boston University. The university's philosophy department had brilliantly taken the initiative of inviting Dirac to explain for the first time how projective geometry had influenced his early work on quantum mechanics. And Roger Penrose, the eminent mathematician and scientist, was the perfect choice as the seminar's moderator. If anyone could prise the story out of Dirac, it was Penrose.

But after giving a short, clear presentation on basic projective geometry, Dirac stopped before connecting it to the quantum was originally discovered by the Arabs. Dirac remarked that his own preference was "strongly on the side of geometry, and has always remained so".

Dirac often said that when he was developing quantum mechanics he used his favourite branch of mathematics - projective geometry - which concerns the relationships between points and straightlines. But why then did he not mention geometry in his early papers?

He expunged his geometrical thinking from his early work, he often said in the 1970 s, because it was especially expensive in those days for journals to print diagrams and because he thought most physicists were unfamiliar with projective geometry. Only later, when John von Neumann invented 'state vectors' did the geometric content of quantum mechanics become plain.

So what were the geometric origins of Dirac's quantum mechanics? I believe that the distinguished French historian of science Olivier Darrigol was the first to set out in detail a plausible explanation. Hepointed out that Dirac had his quantum epiphany when he came across an embarrassed orld. So once Dirac had answered a few straightforward questions, the disappointed Penrose gently turned to him and asked him point-blank how this geometry talk had influenced his early quantum work. Dirac gave his trademark shake of the head, and declined to speak. Realizing that he was on a hiding to nothing, Penrose had no choice but to fill in the time by extemporizing a short talk on a different subject.

Dirac died 12 years later, still having never clarified the point. Perhaps it was beyond clarification? Perhaps even Dirac did not fully understand the connection between his private geometry and his public algebra? As Ludwig Wittgenstein insisted at theend of his Tractatus Logico-Philosophicus, "Whereof one cannot speak, thereof one must remain silent."

Graham Farmelo is Senior Research Fellow at the Science Museum, and Adjunct Professor of Physics at Nor theastern University, Boston, USA.

\section{FURTHER READING}

Galison, P. The Suppressed Deawing Paul Dirac's Hiddan Geometry 145-166 (Univ. California Press, 2000) Darrigd, O. in From c-numbers to q-numbers Ch. 6 (Univ.California Press, 1992) 